\title{
Federal Funding for Mathematics Research
}

\section{Reza Malek-Madani and Karen Saxe}

The AMS Office of Government Relations, located in Washington, DC, works with the federal government to try to make sure that the agencies that award money to mathematicians have the budget they need to ensure robust and stable funding for mathematics research. For example, the Office works with the Coalition for National Science Funding (CNSF) on annual appropriations for the National Science Foundation (NSF), and with the Coalition for National Security Research (CNSR) for Department of Defense (DoD) appropriations. Fair warning: this article contains (too) many acronyms.

Mathematicians working in academia often seek federal funding for their research from the NSF. This makes sense, as about $64 \%$ of federal support for basic research in the mathematical sciences-and done at colleges and universities-comes from the NSF (Figure 1). ${ }^{1}$ NSF funding is available for a wide variety of projects, including for individual investigator awards.

The NSF is the only agency that supports mathematics research broadly across all fields. The goal of this article is

Reza Malek-Madani is a Professor of Mathematics at the US Naval Academy and the Program Officer of the Applied and Computational Program at the Office of Naval Research. He is also a Notices associate edtior. His email address is rmm@usna. edu. Karen Saxe is Associate Executive Director of the AMS and Director of the Office of Government Relations. Her email address is kxs@ams. org.

Opinions expressed in this paper represent the authors' views and not those of the Department of the Navy, or of the American Mathematical Society.

The authors would like to thank the following for help with this article: Fariba Fahroo (Air Force Office of Scientific Research, DoD), James Gimlett (Defense Advanced Research Projects Agency, DoD), Joseph Meyers (Army Research Office, DoD), Haluk Resat (National Institute of General Medical Sciences, NIH), Charles Toll (NSA), and Donald Wagner (Office of Naval Research, DoD).

${ }^{1}$ https: //www.nsf.gov/homepagefundingandsupport.jsp For permission to reprint this article, please contact: reprint -permission@ams.org.

DOI: https://dx.doi.org/10.1090/noti 1858 to explore other federal agency sources of funding for mathematics. The chart below shows that mathematicians are more dependent on the NSF for support than are scientific researchers in some other disciplines. It is worthwhile to note that other agencies contribute funds for mathematics research, but only to projects that contribute to their respective missions.

While $64 \%$ is clearly the majority of funding, this leaves a sizable chunk. Where does the remainder come from? The vast majority of the remainder comes from DoD and the Department of Health and Human Services (HHS) at $18 \%$ and $12 \%$ respectively. The Department of Energy (DoE) provides a smaller portion of funds for mathemati-

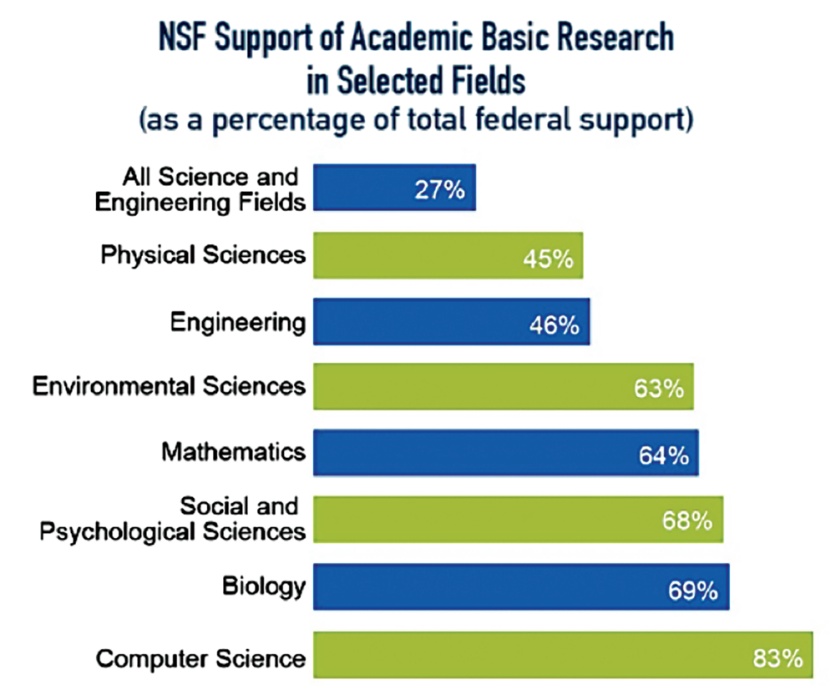

Notes: Biology includes Biological Sciences and Environmental Biology. Biology and Psychological Sciences exclude National Institutes of Health funding from the total amount of federal support.

Source: NSF/National Center for Science and Engineering Statistics, Survey of Federal Funds for Research and Development, FY 2015.

Figure 1. About $64 \%$ of federal support for basic research in the mathematical sciences-and done at colleges and universities - comes from the NSF. 
cal sciences research; this amounts to just under 5\% of all federal funding for basic research done at universities and colleges in mathematics. ${ }^{2}$

The rest of this article will focus on DoD and HHS opportunities for mathematicians. The NSF is the only agency of the federal government that does not do research in its own labs ("in house" or intramural research). DoD and HHS opportunities are thus in the form of grants for faculty members (extramural) and also include options to work at the agency doing intramural research. In this article, we focus on extramural opportunities.

At DoD there are several agencies that contribute significantly to research funding-the Air Force Office of Scientific Research, Army Research Office, Office of Naval Research, Defense Advanced Research Projects Agency, and the National Security Agency.

At HHS, mathematics research is supported by several of the National Institutes of Health's (NIH) 21 institutes, including the National Heart, Lung, and Blood Institute (established 1948), the National Institute of General Medical Sciences (established 1962), and the National Institute of Biomedical Imaging and Bioengineering (established 2000). ${ }^{3}$

\section{Department of Defense funding for mathematics}

Industry is a vital partner for DoD in technology development; it is perhaps not as well known that academic researchers also play a significant role in furthering DoD's mission. According to NSF data, nearly one in every four dollars of DoD awards for scientific research goes to colleges, universities, and non-profits. Further, over $57 \%$ of DoD-sponsored basic research takes place at non-profits, and on college and university campuses. ${ }^{4}$

You may wonder why DoD supports basic research. The mission of the DoD is to provide the military forces needed to deter war and to protect the security of our nation, and DoD's capability to do so depends on technology. Technology is the fruit of science, and basic research produces the new, transcendent ideas that will enable our future technologies. It is the role of DoD Basic Research Program Managers to make informed, but not guaranteed, investments in particular directions that they deem have the best chance of helping provide DoD with the new scientific understanding that will enable unprecedented future technologies.

We highlight five agencies within the DoD. Three are the "services" - Air Force, Army, and Navy. Each of the services has a Young Investigator Program (YIP), and

\footnotetext{
${ }^{2}$ Funding percentages for FY2015. See https://ncsesdata.nsf .gov/fedfunds/2015/htm1/FFS2015_DST_080.htm1

${ }^{3}$ https://www.nih.gov/institutes-nih/1ist-nih -institutes-centers-offices

${ }^{4}$ https://ncsesdata.nsf.gov/fedfunds/2015/
}

their own Multidisciplinary University Research Initiative (MURI). The first of these supports early career scientists who show exceptional promise in the specific service's priority research areas. The awards are generous, and may be budgeted against any reasonable costs related to the conduct of the proposed research, including salary for the young investigator, graduate student support, supplies and operating expenses. Recent YIP awardees include:

- Shayan Oveis Gharan, University Of Washington, "Applications of Algebraic Techniques in Algorithm Design" (Navy 2018);

- Jonathan Hauenstein, University of Notre Dame, "The Geometry of Multiscale Models: Identifiability, Reparameterization, Comparisons, and Parameter Space Exploration" (Army 2014);

- Laura Balzano, University of Michigan, "Non-convex Optimization Algorithms and Theory for Matrix Factorization with Dynamic Massive Data" (Air Force 2018);

- Sanjam Garg, University of California, Berkeley, "Cryptography for Big Data" (Air Force 2017).

Each year there are twenty-four MURI topics, with eight provided by each of the Air Force, Army, and Navy basic research offices. Many of the 2019 topics across all three services seek mathematical models and computational approaches to provide theory and understanding to complex physical and biological processes. As an example, one topic offered by Navy is "Fundamental Limits on Information Latency," and this MURI will require researchers with backgrounds in signal processing, optimization, and game theory. "Advanced Analytical and Computational Modeling of Arctic Sea Ice" is another Navy topic, and the Army has a call out for proposals related to "Multi-layer Network Modeling of Plant and Pollen Distribution across Space and Time." Watch for the 2020 topics announcement.

The strategy of the Air Force Office of Scientific Research (AFOSR) is to invest in basic research with the goal of transferring its fruits to industry, to the academic community to further scientific knowledge, and to the various branches of the Air Force Research Laboratory, for further research and development of technology. Investment in pure mathematics research has been a key ingredient in AFOSR's strategy where funding has been provided primarily in three programs: Dynamics \& Control, Information Science, and Cybersecurity. The research topics being funded include: applied category theory, applied algebraic topology, and analysis. In this context, the word "applied" means seeking new mathematical concepts and theories that have potential applications in scientific disciplines such as dynamics of abstract systems, homotopy type theory, univalent foundations, and formalized proofs.

Since 2011, AFOSR has sponsored a number of single-investigator efforts, including foreign researchers, and a MURI project on Homotopy Type Theory and Univalent Foundations. The latter has the participation of a number of 


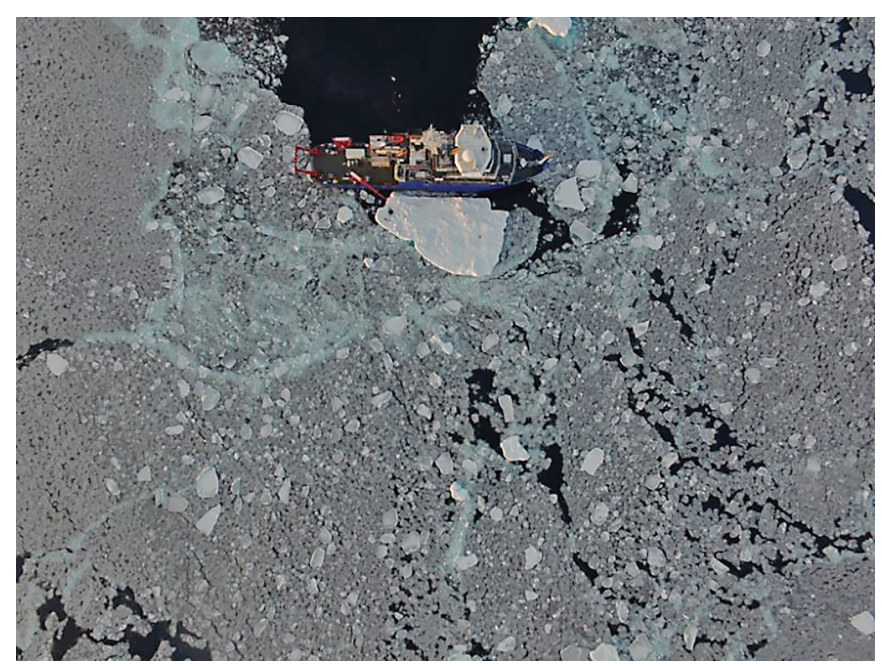

Figure 2. A snapshot of the state of sea ice in the Arctic, displaying its complex multiscale nature.

mathematicians, including Vladimir Voevodsky (deceased; formerly at the Institute for Advanced Study), Michael Shulman (University of San Diego), and Steve Awodey and Jeremy Avigad (both at Carnegie Mellon University). AFOSR has also sponsored work on data interpolation since 2011. In this effort, mathematicians are collaborating with control theorists to formulate a new framework for modeling and learning dynamical systems using only small amounts of data. Sponsorship of research on using category theory and operads to study abstract complex systems and their dynamics is leading to new discoveries and directions in this field. A group of mathematicians are using Wasserstein geometry and optimal transport for computer vision problems. AFOSR has sponsored research at the intersection of systems biology and differential equations. Another sponsored effort is investigating robotic navigation via sheaf-theoretic techniques.

In applied and computational mathematics, AFOSR has had several MURIs in areas such as Uncertainty Quantification (combination of applied probability and computational tools), Game Theory on Networks, and most recently in 2018 a MURI on Mean-Field Game Theory.

For more information: https://www.wpafb . af.mi1/afr1/afosr.

The Army Research Office supports any sort of mathematics that may help enable a stronger future Army, and is the only funding agency within the DoD with a Mathematical Sciences Division. This division is currently organized into four programs: Modeling of Complex Systems (interests involving geometry, topology, non-smooth differential analysis), Probability and Statistics (interests involving SDEs, QSDEs, multivariate heavy-tailed phenomena), Biomathematics, and Computational Mathematics.

In addition to the YIP and MURIs described above, Army funding can be obtained through several programs, including: Single Investigator program, Short-Term Innovative
Research (STIR) program, Presidential Early Career Award for Scientists and Engineers (PECASE), Historically Black College and Universities/Minority Institutions (HBCU/ MI) program, Conference Grants, and the High School Apprenticeship Program (HSAP)/Undergraduate Research Apprenticeship Program (URAP). This agency supported John Tukey's work in statistical analysis, and his famous Fast Fourier Transform paper with Cooley acknowledges the Army Research Office as sole sponsor. ${ }^{5}$ They also supported Lotfi Zadeh's work in fuzzy mathematics, which was honored posthumously with a 2017 Golden Goose Award. ${ }^{6}$

For more information: https: //www.ar 1 . army . mi 1 .

The Office of Naval Research (ONR) funding opportunities are organized according to technology needs; mathematics falls in the department of "Command, Control, Communications, Computers, Intelligence, Surveillance, and Reconnaissance" (or, "Code 31 "). ${ }^{7}$ Code 31 is further broken down into three divisions, one of which is "Mathematics, Computer and Information Systems" (Code 311). Code 311 funds basic research in areas including network theory, decision-making, cybersecurity, mathematical data science, and computational analysis. It includes three programs that have significant mathematical content.

- The Applied and Computational Mathematics Program focuses on developing analytical and computational tools for solving PDEs arising from various physical problems, such as ocean and atmospheric dynamics, inverse problems in acoustics, imaging targets in cluttered media, and multi-scale/multi-physics problems arising in the modeling of fatigue, fractures, and shocks. The mathematical modeling of sea ice dynamics and its multiscale nature is a current focus of research and several applied mathematicians are working on this topic (see Figure 2).

- The Mathematical Data Science Program focuses on developing mathematical tools for efficiently representing data, understanding relationships in data, and extracting information from data. This program draws upon basic research in mathematics, probability, statistics, signal processing, machine learning, data engineering, and information theory.

- The Mathematical Optimization Program focuses on developing theory and algorithms for solving largescale optimization problems. This includes, but is not limited to, cutting plane and polyhedral techniques for mixed-integer programming, decomposition ap-

\footnotetext{
${ }^{5}$ Cooley, J. W. and Tukey, J. W. "An Algorithm for the Machine Calculation of Complex Fourier Series." Math. Comput. 19, 297-301, 1965.

6 https://www.goldengooseaward.org/awardees/fuzzy -logic

7https: //www. onr.navy.mi1/en/Science-Technology /Departments/Code-31
} 
proaches for large convex and non-convex problems, first- and second-order algorithms for convex optimization, and distributionally robust methods for stochastic optimization. Also of interest is research in combinatorial optimization and discrete structures associated with these problems.

There are additional programs within the Division in which mathematics plays a supporting role, such as the Machine Learning, Reasoning, and Intelligence Program, and the Cyber Security and Complex Software Systems Program.

ONR-supported research has led to the proofs of the Four Color Theorem, the Strong Perfect Graph Theorem in graph theory, and Rota's Conjecture in matroid theory.

The Naval Research Enterprise (which includes ONR and the Naval Research Laboratories) has a variety of opportunities for students (undergraduate and graduate) and faculty. ${ }^{8}$

For more information: https://www. on r. navy . mi 1 .

The Defense Advanced Research Projects Agency (DARPA) funds projects focused on improving our national security. Many efforts across the agency may have a significant mathematical component, and within the Defense Sciences Office (DSO) of DARPA, several programs have had a strong mathematical focus. Mathematics-focused programs are generally aimed at developing new mathematical approaches and algorithms to solve problems spanning Artificial Intelligence, optimization, uncertainty quantification, dynamical systems modeling, control theory and design. Indeed the current DSO Broad Agency Announcements (BAAs) list "Frontiers in Math, Computation and Design" as one of four research topics of interest and will support basic research that fits with the call for proposals. ${ }^{9}$ It also offers a Young Faculty Award program to identify and engage rising research stars in junior faculty positions at US academic institutions. ${ }^{10}$ Mechanical engineer Mark Fuge of the University of Maryland is a Young Faculty awardee for a project entitled "Topology and Synthesis of Design Manifolds," studying how mathematical tools from topology, group theory, and machine learning can aid human exploration of large, complex design spaces.

For more information: https://www. darpa . mi 1.

The National Security Agency's Mathematical Sciences Program (MSP) was "started in 1987 in response to an increasingly urgent need to support mathematics in the United States." 11 The MSP supports conferences and

\footnotetext{
$\overline{8 \text { https://www.onr.navy.mi1/Education-Outreach }}$

${ }^{9}$ https: / /www . fbo.gov/index?s=opportunity\& $\operatorname{mode}=$ form\&id=36a2f123679010655 f4930a $24 \mathrm{cc}$ $8 a 750 \& t a b=$ core\&_cvi ew $=0$

${ }^{10}$ https: //www. darpa.mi1/work-with-us/for-universities/young-faculty-award

${ }^{11}$ https: //www.nsa.gov/what-we-do/research /math-sciences-program
}

workshops in the five subject areas of Algebra, Discrete Mathematics, Number Theory, Probability, and Statistics. They also support Research Experiences for Undergraduates programs in any area of mathematics or computer science. In the past, MSP has funded individual researchers. For budgetary reasons, individual grants as well as Young Investigator Grants and the NSA Sabbatical Program are not currently available. It is possible that we will see these opportunities re-emerge in the future.

For more information: https://www.nsa.gov.

In addition to the opportunities described above, there are joint programs between the NSF and other agencies. The "Algorithms for Threat Detection" program is just one example, and is jointly run by NSF and DoD. Harvard mathematician Shing-Tung Yau currently holds an award and is using tools from random graph theory and differential geometry to focus on finding patterns in large graphs that may be hidden and that could potentially be indicative of emerging threats of various kinds (internets, critical infrastructure networks, financial networks, social networks, etc.).

\section{Department of Health and Human Services funding for mathematics}

As with all other federal agencies outside of the NSF, research supported by HHS is mission driven. The NIH sits in HHS and, as you probably know, it works to prevent disease and improve health. Mathematicians have an array of opportunities to collaborate through NIH funding. Reinhard Laubenbacher of the University of Connecticut is working with a medical colleague on algorithms that could lead to new therapies to treat invasive Aspergillosis, a fungal disease of the lungs that poses serious health dangers to patients with weakened immune systems, including organ transplant patients and cancer patients undergoing chemotherapy.

Within the National Institute of General Medical Sciences at NIH, the Division of Biophysics, Biomedical Technology, and Computational Biosciences supports research in mathematical biology through a program run jointly with the NSF. ${ }^{12}$ Both agencies recognize the need for promoting research at the interface between the mathematical sciences and the life sciences. This program is designed to encourage new collaborations, as well as to support existing ones. Mathematician Christine Heitsch (Georgia Tech), whose research interests lie at the interface between discrete mathematics and molecular biology, has been supported by this program.

The National Institute of Biomedical Imaging and

\footnotetext{
12 https://www.nigms.nih.gov/Research/specificareas /mathbio/Pages/default.aspx
} 


\section{COMMUNICATION}

Bioengineering funds research in modeling, simulation, and analysis. ${ }^{13}$ As with DoD, the NIH also collaborates with the NSF and there are inter-agency programs such as the Collaborative Research in Computational Neuroscience. ${ }^{14}$ For more information: https://www.nih.gov.

\section{Summary}

Federal funding for mathematical research is available from agencies other than the NSF. The DoD and NIH granting agencies, in tandem with the NSF, have had a remarkable record of focused investments in almost all areas of mathematics, but especially in applied and computational mathematics, that have resulted in rapid acceleration of several areas with game-changing effects on science and technology. Among these achievements are algorithm developments ranging from the Simplex method to the Fast Multipole method, the development of wavelets and its huge impact on JPEG 2000, to compressive sensing, uncertainty quantification, and now the mathematics of mean fields. All of these developments have benefited from investments by the NSF and the DoD and NIH granting agencies.

This article has attempted to give an overview of funding opportunities at the DoD and the NIH, in particular. Key advice for the potential PI:

(a) Keep an eye on Broad Agency Announcements, the announcements that the DoD and NIH granting offices routinely publish to communicate launching of new initiatives.

(b) Have a conversation with the program officer, who often has a large degree of autonomy in developing their research portfolio, prior to submitting a proposal or a white paper to determine whether the proposed research is a good fit for the program.

(c) Remember that both the DoD and NIH are charged with developing a portfolio of research investments that contribute to their respective missions.

\footnotetext{
13https://www.nibib.nih.gov/research-funding /mathematical-modeling-simulation-and-analysis ${ }^{14}$ https: //www.nsf.gov/funding/pgm_summ.jsp?pims $-i d=5147$
}
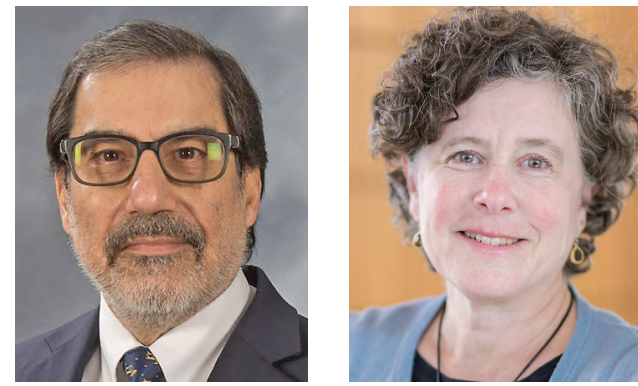

Reza Malek-Madani Karen Saxe

\section{Credits}

Figure 2 is courtesy of Guy Williams (University of Tasmania/ ACE CRC) and Ted Maksym (WHOI).

Photo of Reza Malek-Madani is courtesy of the Photo Lab: US Naval Academy.

Photo of Karen Saxe is courtesy of Macalester College/David Turner.

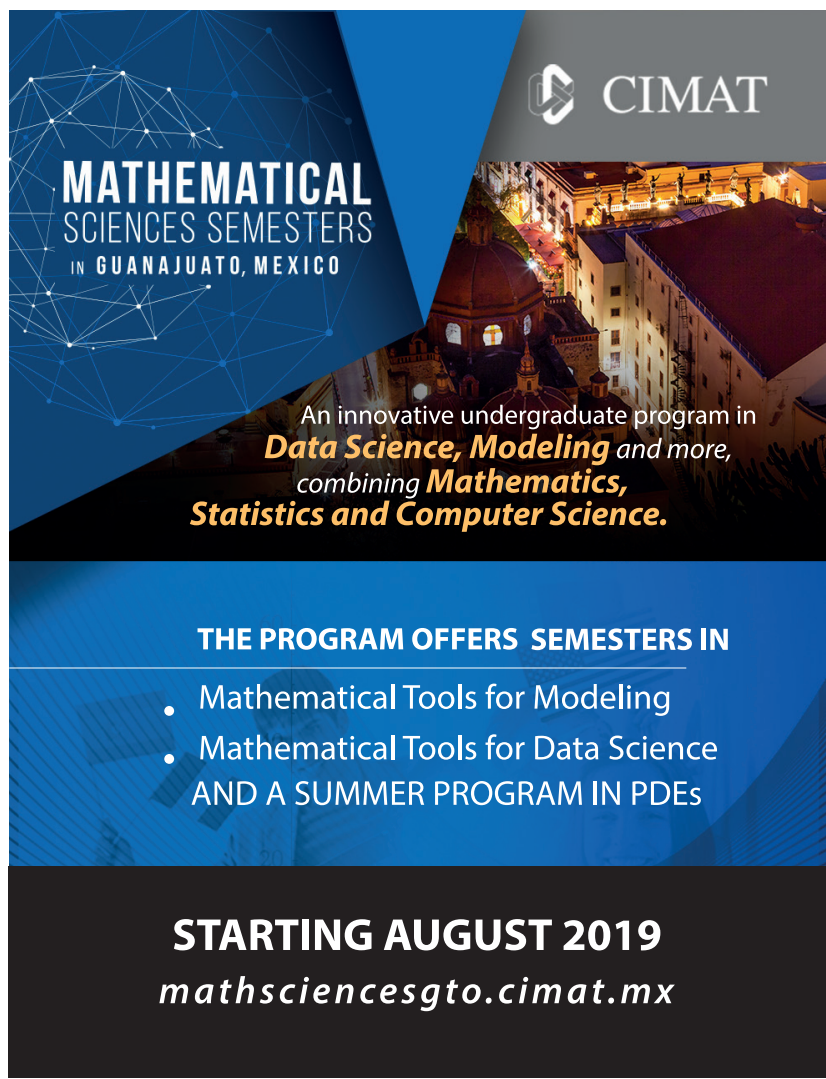

